\title{
Increased Bone Turnover with Decreased Bone Formation by Osteoblasts in Children with Osteogenesis Imperfecta Tarda
}

\author{
ROLAND BARON, ${ }^{(34)}$ JOSEPH M. GERTNER, ROBERT LANG, AND AGNES VIGNERY
}

Yale University School of Medicine, Departments of Internal Medicine and Pediatrics, New Haven, Connecticut, USA

\begin{abstract}
Summary
Iliac crest bone biopsies from nine children (6-15 yrs old) with osteogenesis imperfecta tarda (OI) have been studied by bone histomorphometry after double fluorescent labeling with tetracycline and compared to five unlabeled biopsies from normal children in the same age group. The results indicate that children with $O I$ have a low trabecular bone volume associated with an increased bone turnover rate. Bone formation is increased at the tissue level despite a decrease in the activity of individual osteoblasts. The original defects in $\mathrm{OI}$ seems, therefore, to be in the rate of matrix synthesis by osteoblasts. It is, however, compensated by an increase in the number of these cells. These results suggest that these children were not losing bone at the time of the biopsy, which fits with the clinical stability of OI with age. Our study therefore suggests that the osteopenia observed in OI is most likely due to an inability to accumulate bone during growth, as normal children do, rather than to a progressive net loss of bone.
\end{abstract}

\section{Abbreviations}

OI, osteogenesis imperfecta tarda

MWT, mean wall thickness

TBV, trabecular bone volume

The high fracture rates in children with osteogenesis imperfecta tarda is associated with both a decreased amount of bone $(7,29$, $30)$ and an abnormal bone matrix $(4,11,13,33)$. The pathogenesis of the classically observed osteopenia is not well understood. It has been alternatively attributed either to decreased bone formation $(15,27,29)$, with or without decreased resorption $(28)$, or to increased bone resorption, with or without decreased formation $(7,14,26)$. The purpose of this study was therefore to measure bone remodeling by dynamic histomorphometry of iliac crest biopsies in children with osteogenesis imperfecta tarda and compare it to normal controls. The results indicate the presence of an increased rate of bone turnover with a decreased rate of bone matrix synthesis by the osteoblasts. Because both the coupling and the balance between resorption and formation appear to be maintained, the pathogenesis of the osteopenia in osteogenesis imperfecta tarda (OI) is therefore probably due to an uncompensated transient bone loss or, more likely, an inability to accumulate bone with age in childhood rather than to a permanent negative balance that would lead to a total loss of trabecular bone over the course of this hereditary disease. We define coupling as the qualitative succession of bone formation and resorption at the same bone remodeling site and balance as the quantitative equilibrium between these two activities at the tissue level (2).

\section{MATERIALS AND METHODS}

Nine children with OI were selected for this study by the presence of three clinical criteria: history of fractures, blue sclerae, and positive family history. There were seven females and two males of an average age of 10 years, ranging from 6-15. Clinical details are given in Table 1. Each patient was given oral oxytetracycline (Terramycin) at $10 \mathrm{mg} / \mathrm{kg}$ of body weight in three doses per day on days 16 and 15 and days 4,3 , and 2 before biopsy. A transcortical iliac crest biopsy was then performed under local anesthesia $2 \mathrm{~cm}$ inferior and $2 \mathrm{~cm}$ posterior to the anterosuperior iliac spine. The biopsies were fixed in $40 \%$ ethanol at $4^{\circ} \mathrm{C}$, dehydrated through graded alcohols and embedded without decalcification in methyl-methacrylate (3). Sections, 4 microns thick, were obtained with a Jung $\mathrm{K}$ sliding microtome and stained with Toluidine blue ( $\mathrm{pH} 3$ ), Von Kossa or Goldner Trichrome. Sections 8-10 microns thick were kept unstained for fluorescence microscopy. Biopsy material from the same anatomical area was obtained from five normal children in the same age range (two acute accident victims and three during surgery for congenital hip displacement) and processed the same way with the exception that they were not labeled with tetracycline. Histomorphometric measurements were made using a planimeter (MOP 3, Zeiss, Germany), as previously described (6). Calculations were made according to Frost (18) and corrected for obliqueness of sections when appropriate $(\times 0.73)$. Statistical comparison between the two groups were made by using nonparametric tests for small samples (MannWhitney, Median test) and family regression. The differences were considered as significant for a $P$ value lower than or equal to 0.05 .

Biochemical studies. After 3 days on a low $(400 \mathrm{mg})$ calcium and hydroxyproline restricted diet, a fasting blood sample was obtained and a 24-h urine collected while the patients were fully ambulant. Blood urea nitrogen, serum creatinine, electrolytes, calcium, magnesium, phosphorus, and alkaline phosphatase, as well as urine creatinine, calcium and phosphorus, were measured by routine methods. Serum parathyroid hormone was measured by radioimmunoassay by the Nichols Institute, San Pedro, CA using a guinea pig antibody (20). 25-Hydroxyvitamin D was measured by direct ultraviolet absorbance after high pressure liquid chromatography on plasma extracts that had previously been run over Sephadex LH20 and Lipidex columns (32). Urinary hydroxyproline was measured by the method of Parekh and Jung (25).

\section{RESULTS}

Bone morphometry, Static Parameters (Table 2). The overall trabecular bone volume (TBV) was significantly lower in the OI group than in the control group; however one female, 9 years old, had a normal value for TBV (27.3\%) and three others had only a moderately decreased TBV (19-22\%). In parallel, mean trabecular width was significantly decreased in the OI group. Static parameters of bone formation showed a normal absolute volume of osteoid but, because of the low TBV, an increase in the volume of osteoid relative to trabecular bone. This relative increase was associated with a marked and significant increase in osteoid surface extent and a proportional increase in the osteoblastic 
Table 1. Clinical data

\begin{tabular}{cccccc}
\hline Patient & Sex & Age (yr) & $\begin{array}{c}\text { Family } \\
\text { history }\end{array}$ & $\begin{array}{c}\text { Degree of } \\
\text { incapacity }\end{array}$ & $\begin{array}{c}\text { Blueness }^{3} \\
\text { of sclerae }\end{array}$ \\
\hline 1 & F & 6.4 & Yes & 1 & 2 \\
2 & F & 11.0 & Yes & 1 & 2 \\
3 & F & 11.2 & Yes & 5 & 2 \\
4 & F & 7.1 & Yes & 0 & 3 \\
5 & M & 6.3 & Yes & 0 & 3 \\
6 & M & 14.1 & No & 0 & 3 \\
7 & F & 12.9 & No & 4 & 2 \\
8 & F & 14.6 & No & 3 & 2 \\
9 & F & 9.3 & No & 0 & 1 \\
\hline
\end{tabular}

'Compatible with dominant inheritance in all positive cases.

${ }^{2} 0$, no incapacity and 5 , unable to walk, wheelchair-bound.

${ }^{3}$ Arbitrary scale: 0 , white to 5 , deep blue.

Table 2. Bone morphometry: static parameters of bone remodeling, mean $(S . D .)^{1}$

\begin{tabular}{lccc}
\multicolumn{4}{c}{ remodeling, mean $($ S. $D)}$. \\
& $\begin{array}{c}\text { Normals } \\
(n=5)\end{array}$ & $\begin{array}{c}\text { Osteogenesis } \\
\text { imperfecta } \\
(n=9)\end{array}$ & Difference \\
\hline TBV $(\%)$ & $25.5(2)$ & $17.1(6.1)$ & $<0.05$ \\
TW $(\mu)$ & $204(44)$ & $152(38)$ & $<0.05$ \\
AOV $(\%)$ & $0.7(0.1)$ & $1.0(0.6)$ & $\mathrm{NS}$ \\
ROV $(\%)$ & $2.5(0.3)$ & $5.5(1.6)$ & $<0.02$ \\
OS $(\%)$ & $21.6(2.4)$ & $48.5(10.0)$ & $<0.02$ \\
OT $(\mu)$ & $11.4(0.4)$ & $8.9(4.1)$ & $<0.05$ \\
ObS $(\%)$ & $13.9(1.0)$ & $26.3(13.3)$ & $<0.02$ \\
OCS $(\%)$ & $1.1(0.3)$ & $2.0(1.1)$ & $<0.02$ \\
Rev S $(\%)$ & $5.9(2.3)$ & $6.6(1.5)$ & $\mathrm{NS}$ \\
TLS $(\%)$ & $7.0(2.5)$ & $8.7(2.1)$ & $<0.05$ \\
\# OC $/ \mathrm{mm}$ & $0.12(0.02)$ & $0.30(0.16)$ & $<0.05$ \\
MWT $(\mu)$ & $44.7(3.6)$ & $34.8(6.5)$ & $<0.05$ \\
\hline
\end{tabular}

'Abbreviations used in this Table: TBV, trabecular bone volume, $\%$ of whole tissue; TW, mean trabecular width; AOV, absolute osteoid volume, $\%$ of whole tissue; ROV, relative osteoid volume, $\%$ of trabecular bone tissue; OS, osteoid surface, \% of total trabecular bone surface; OT, mean osteoid seam thickness; ObS, active osteoblastic surface, $\%$ of total bone surface; OCS, osteoclastic surface, \% of total trabecular bone surface; Rev $\mathrm{S}$, reversal surface (nonosteoclastic Howship's lacunae), \% of total trabecular bone surface; TLS, total lacunar surface, \% of total trabecular bone surface; \# OC/mm, number of osteoclasts per $\mathrm{mm}$ of trabecular bone perimeter; and MWT, mean wall thickness (average thickness of completed remodeling units).

surface. The average thickness of osteoid seams was however significantly lower than normal. There was therefore an overall increase in static parameters of bone formation as compared with the normal group. In parallel, a significant increase in all the static parameters of bone resorption (number of osteoclasts, osteoclastic surface, total lacunar surface) was observed but there was no accumulation of reversal lacunae, therefore excluding an uncoupling between resorption and formation. The mean wall thickness (MWT) of completed remodeling units was lower than in the control group.

Bone morphometry, dynamic parameters (Table 3). Our control group not being labeled the dynamic parameters can only be compared with data published in the literature and our own results from children with other types of diseases $(1,6)$. The extent of tetracycline-labeled surface relative to the osteoid surface was normal, as well as the extent of the double labeled surface relative to the osteoblastic surface, therefore showing a normal coupling formation-mineralization. But the mineralization rate seemed to be low for such an age group. Despite this decreased mineralization rate $(\times 0.77)$ and because of the markedly increased formation surface $(\times 2)$, the bone formation rate was high at the tissue level $(\times 1.54)$. Given the low TBV, this led to a high bone turnover rate (65\%/year). Inasmuch as the decreased mineralization rate is associated with a proportionally decreased osteoid seam thickness $(\times 0.73)$, the rate of matrix synthesis had to be low at the osteoblast level and at each individual remodeling site.

There was a significant correlation between the bone formation rate at the tissue level with the TBV $(r=0.65, P<0.05)$ and with the osteoclastic surface $(r=0.87, P<0.01)$, both indicating a good coupling between resorption and formation (Fig. 1). Bone formation rates calculated by adding half of the single labeled surface are also given (Table 3 ).

Bone morphometry, biochemical studies (Table 4). The only biochemical parameter that has been found to be slightly abnor$\mathrm{mal}$ in this group of patients with OI was the urinary hydroxyproline excretion expressed as a ratio to creatinine. Twenty-four hour hydroxyproline excretion was above the mean for this age $(8,21)$ in eight of the nine patients. In four of them, the hydroxyproline excretion was more than 2 standard deviations above the normal mean value.

The only significant correlations with bone histomorphometric measurements were observed between serum alkaline phosphatase and the bone formation rate at the tissue level $(r=0.75, P<0.02)$

Table 3. Bone morphometry: dynamic parameters of bone remodeling, mean (S.D.)

\begin{tabular}{llc}
\hline \multicolumn{1}{|c}{$\begin{array}{c}\text { Normals } \\
(n=5)\end{array}$} & $\begin{array}{c}\text { Osteogenesis } \\
\text { imperfecta } \\
(n=8)\end{array}$ \\
\hline MR $(\mu /$ day $)$ & $1^{2}$ & $0.65(0.08)$ \\
& $0.85(0.10)^{3}$ & \\
2 labels $(\%)$ & $0.84^{4}$ & \\
1 label $(\%)$ & & $20.0(10.1)$ \\
BFR $\left(\mathrm{mm}^{3} / \mathrm{mm}^{2} / \mathrm{yr}\right)$ & & $20.1(16.4)$ \\
BFR $(\mathrm{BMU})\left(\mathrm{mm}^{3} / \mathrm{mm}^{2} / \mathrm{yr}\right)$ & & $0.07(0.03)$ \\
TOR $(\% / \mathrm{yr})$ & $\sim 40$ & $0.10(0.06)$ \\
\hline
\end{tabular}

'Abbreviations used in this Table: MR, corrected $(\times 0.73)$ mineralization rate; 2 labels, surface with double tetracycline labels, $\%$ of total trabecular bone surface; 1 label, surface with single tetracycline label, \% of total trabecular bone surface; BFR, bone formation rate at the tissue level, $\mathrm{mm}^{3}$ of calcified bone $/ \mathrm{mm}^{2}$ of trabecular bone surface/year (calculated on the basis of double labeled surface + half of the single-labeled surface); BFR (BMU), bone formation rate at the bone remodeling unit level, $\mathrm{mm}^{3}$ of calcified bone $/ \mathrm{mm}^{2}$ of osteoid surface/year; TOR, turnover rate, \% of trabecular bone volume/year.

${ }^{2}$ Normal children (24).

${ }^{3}$ Value from children with chronic renal failure "normalized" after 2 years of treatment with $25(\mathrm{OH}) \mathrm{D}_{3}(1)$.

${ }^{4}$ Value from a child with pycnodysostosis (6).

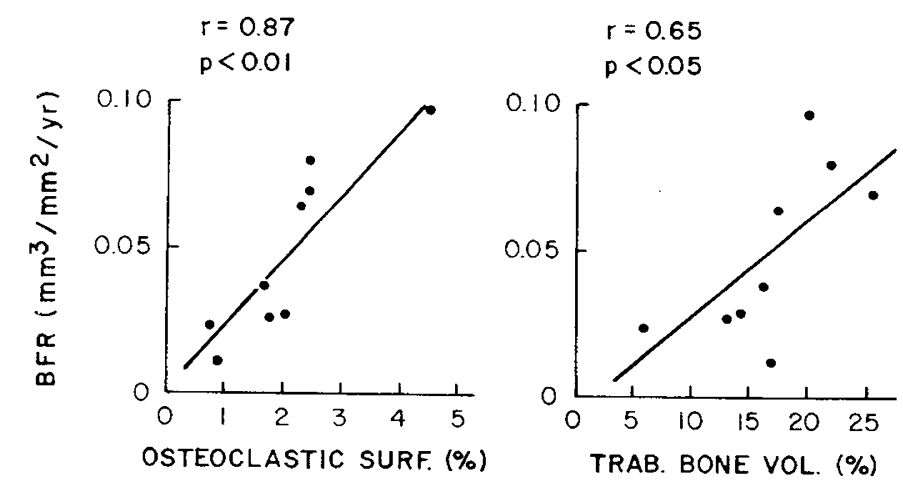

Fig. 1. Correlations between the bone formation rate at the tissue level (BFR, calculated from double labeled surfaces) and osteoclastic surface (left panel) or trabecular bone volume (right panel), demonstrating a good coupling and balance between resorption and formation in nine children with osteogenesis imperfecta. 
Table 4. Biochemical values obtained from individual patients ${ }^{1}$

\begin{tabular}{|c|c|c|c|c|c|c|c|c|c|}
\hline & 1 & 2 & 3 & 4 & 5 & 6 & 7 & 8 & 9 \\
\hline \multicolumn{10}{|l|}{ Serum } \\
\hline Calcium (mg/dl) & 10.4 & 9.6 & 9.6 & 9.9 & 10.1 & 9.8 & 10 & 9.6 & 10.2 \\
\hline Phosphorus (mg/dl) & 5.5 & 5.6 & 4 & 4.5 & 4.9 & 5.1 & 4.5 & 3.8 & 3.8 \\
\hline Magnesium (mg/dl) & 1.5 & 2.2 & & 2.1 & 2 & 2.1 & 2 & 2.3 & 2.2 \\
\hline Alkaline Phosphatase (I.U.) & 85 & 146 & 201 & 189 & 130 & 196 & 116 & 90 & 292 \\
\hline Creatinine $(\mathrm{mg} / \mathrm{dl})$ & 0.4 & 0.4 & 0.7 & 0.5 & 0.5 & 0.5 & $0 . .6$ & 0.6 & 0.4 \\
\hline $\mathrm{iPTH}^{2}(\mathrm{ulEq} / \mathrm{ml})^{*}$ & 78 & 84 & 68 & 93 & 94 & 102 & 91 & 99 & 149 \\
\hline 25 OHD (ng/ml) & 16 & 21 & 12 & 15 & 23 & 8 & 19 & 15 & 10 \\
\hline \multicolumn{10}{|l|}{ Urine } \\
\hline Volume (ml) & 600 & 1080 & 680 & 532 & 419 & 880 & 189 & 315 & 450 \\
\hline Calcium (mg) & 108 & 45 & 7 & 26 & 67 & 107 & 21 & 5 & 188 \\
\hline Phosphorus (mg) & 720 & 848 & $\ldots$ & 367 & 627 & 880 & 167 & 212 & 581 \\
\hline Hydroxyproline (mg) & 67 & 64 & 31 & 42 & 51 & 79 & 23 & 24 & 65 \\
\hline Creatinine (mg) & 465 & 583 & 256 & 430 & 885 & 994 & 129 & 253 & 502 \\
\hline
\end{tabular}

' Normal range (adults), 20-100 $\mu \mathrm{lEq} / \mathrm{ml}$; normal range for alkaline phosphatase is age dependent; other parameters are standard.

${ }^{2}$ iPTH, immunoreactive parathyroid hormone.

and between serum alkaline phosphatase and the osteoblastic surface $(r=0.76, P<0.01)$. No significant correlation could be found between hydroxyproline excretion and bone resorption, alkaline phosphatase or bone turnover rates.

\section{DISCUSSION}

Our results show that children with OI have a decreased TBV associated with an increased bone turnover rate, with increased resorption and formation activities at the tissue level, and a decreased bone formation rate at the level of individual osteoblasts. In other words, a decreased amount of bone matrix is formed by individual osteoblasts and this is compensated by an increase in the number of these cells so that overall, the total amount of bone formed per unit of bone tissue is increased. We interpret these data as indicating the presence of an osteoblastic defect leading to a negative bone balance during a limited period of time, perhaps until the rate of activation of new remodeling units increases and stops this loss of bone. The osteopenia can therefore be explained either as a failure to accumulate bone normally with age in childhood or as the consequence of a temporary negative bone balance that occurred at one time during the course of the disease. The first hypothesis is, however, more likely because no changes have been noticed as a function of age, clinically or morphometrically, here and in other studies $(14,26)$. This osteopenia, associated with possible qualitative defects of the bone matrix $(4,11,13,15,27,28,33)$, is very likely responsible for the high fracture rates observed in such children. No quantitative defects in the mineralization process have been observed in our patients.

The lack of double fluorescent labeled normal children, in this study as well as in others $(5,19,22,23)$ certainly constitutes a main difficulty in the interpretation of the data. It seems, however, essential to point out the following observations, all of which strongly support the fact that bone formation is decreased at the osteoblastic level in our patients. First, double label measurements made in a group of children with chronic renal failure, in the same age range, and which have been shown to be "normalized" by all other criterions at the level of bone after 2 years of treatment with $25(\mathrm{OH}) \mathrm{D}_{3}(1)$ showed a corrected mineralization rate of 0.85 microns per day $( \pm 0.10)$, a value significantly higher than this group of children. Second, we obtained a similar value in one child, age 15 , with pycnodysostosis (6). Third, other values found in the literature for cortical bone $(17,22)$ suggest that children have a much higher mineralization rate than adults. Finally, values obtained recently in a few double labeled normal children by another group seem to confirm that they normally show a mineralization rate of about one micron per day (24). It therefore seems justified to consider that the mineralization rate in these children with OI is decreased. In addition, the fact that these children also show a decreased osteoid seam thickness further demonstrates that the rate at which bone matrix is formed by the osteoblasts is also decreased. Interestingly, the decrease in mineralization rate is quantitatively very similar to the decrease in osteoid seam thickness (about 25\%), confirming a proportional decrease in bone matrix formation. Finally, the significant decrease in the mean wall thickness of each remodeling unit is a strong additional evidence that bone formation rates are decreased at the cellular and remodeling unit levels. In terms of balance however, this is overcompensated by a $100 \%$ increase in the bone forming surfaces, a value strictly parallel to the increase in bone resorbing surfaces.

Ramser et al. (26) reached almost exactly the same conclusions after studying bone dynamics in three women with osteogenesis imperfecta. They used tetracycline labels on rib biopsies and measured bone remodeling activity in the cortical bone. Their results showed an increased turnover rate with high resorption and formation rates at the tissue level but a low formation rate at the bone remodeling unit level and cellular level. We reached the same conclusions here in the trabecular bone of eight children with the same disease. Their interpretation, however, differs from ours in that they suggest that the more time is given to the bone to correct for osteopenia, with increasing age, the lower the fracture rate. It is, however, striking that their patients were age 30,51 , and 70 , respectively and still showed the same changes in bone remodeling, at another site of the skeleton and in cortical instead of trabecular bone, than our group of young children. This might indicate that instead of "catching up," the skeleton is only able to stabilize the amount of bone present in both cortical and trabecular bone, never really correcting for the bone loss that previously occurred. As suggested by Falvo and Bullough (14) this bone loss might only be relative to normal controls and not absolute: normal children seem to increase their bone volume with age $(14,24)$ and children with OI might lack the ability to accumulate bone rather than actually loosing bone. It is not possible, from our study or the above mentioned ones to know which of these two hypotheses is true, i.e., a transient negative bone balance corrected by an increase in the number of osteoblasts or a lack of positive balance at a time when normal children increase their bone volume. In both cases our observations would explain why, despite an equilibrium between bone resorption and formation, both at increased rates, patients with OI are osteopenic. This concept is very important in that it suggests that no negative balance is occurring in these patients at the time of the biopsy, therefore explaining the constant, although low, bone volume throughout the life of these patients.

Altogether, our results point toward an abnormal osteoblastic function at the cellular level, a conclusion previously reached by Ramser et al. (26) and Falvo and Bullough (14) from morphometric studies of bone biopsies and by Doty and Mathews (12) at the electron microscopic level. These latter authors have shown that the osteoblasts of patients with OI had large accumulations 
of glycogen in their cytoplasm and a decreased amount of alkaline phosphatase and adenosine monophosphatase in their membrane, as demonstrated by enzyme histochemistry. Both observations suggest a decreased synthetic activity. The ultrastructure and enzymatic activity of osteoclasts and osteocytes was found to be normal. It also seems clear from other studies that not only is the amount of bone matrix formed by each osteoblast decreased, but both its collagenous and noncollagenous components are abnormal $(11,28,30)$. The part played by these qualitative abnormalities versus the quantitative lack of bone in the incidence of fractures is not yet established.

Finally, the observation of a high bone turnover rate fits very well with the increased hydroxyproline excretion, an observation frequently made in these patients $(9,12)$.

In conclusion, although children with OI are osteopenic, it seems unlikely that they are in an ongoing negative bone balance. The primary defect, which seems to be that the osteoblasts are unable to synthesize a normal amount of bone matrix, is compensated by an increase in the number of these cells, leading to a high turnover rate. It is suggested that their osteopenia most likely results from an inability to accumulate bone during the growth period, as normal children do $(10,17)$ or from a transient negative bone balance occurring at one time during the course of the disease but this has never been observed clinically.

\section{REFERENCES AND NOTES}

1. Baron, R., Norman, M., Mazur, A., Gruskin, A., and Rasmussen, H. Bone histomorphometry in children with early chronic renal failure treated with

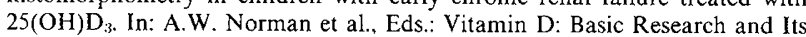
Clinical Application. pp. 847-850 (W. deGruyter, New York, NY, 1979).

2. Baron, R., Vignery, A., and Lang, R.: Reversal phase and osteoporosis: Defective coupling of resorption to formation in the pathogenesis of osteoporosis. In: H.F. DeLuca et al., Eds.: Osteoporosis: Recent Advances in Pathogenesis and Treatment. pp. 311-320 (University Park Press, Baltimore, MD, 1981).

3. Baron, R., Vignery, A., Neff, L., Silverg!ate, A., and Santa Maria, A.: Processing of undecalcified bone specimens for bone histomorphometry. In: R. Recker Ed. Bone Histomorphometry: Techniques and Interpretation. in press (CRC Press, Baltimore, MD, 1982)

4. Brown, D. M.: Biochemical abnormalities in osteogenesis imperfecta. Clin. Orthop. Rel. Res., 159: 75 (1981).

5. Bulla, M., Delling, G., Offerman, G., Ziegler, R., Benz, G., Luehmann, H., and deReutter, A. S.: Renal bone disorder in children. In: A.W. Norman et al., Eds.: Vitamin D: Basic Research and Its Clinical Application. pp. 853-859 (W. deGruyter, New York, NY, 1979).

6. Canalis, E., Reardon, G. E., Baron, R., and Raisz, L. G.: Dynamic bone morphometry and studies on the effects of serum on bone metabolism in vitro in a case of pycnodysostosis. Metab. Bone Dis. Rel. Res., 2: 99 (1980).

7. Castells, S., Colbert, C., Chakrabarti, C., Bachtell, R. S., Kassner, E. G., and Yasamura, S.: Therapy of osteogenesis imperfecta with synthetic salmon calcitonin. J. Pediatr., 95: 807 (1979).

8. Clark, S. and Zorab, P. A.: Hydroxyproline centiles for normal adolescent boys and girls. Clin. Orthop. Rel. Res., 137: 217 (1978).

9. Cropp, G. T. A. and Myers, D. N.: Physiological evidence of hypermetabolism in osteogenesis imperfecta. Pediatrics, 49: 375 (1972)

10. deGruembecker, W., deGruembecker, C., and Duriez, J.: Variation du contenu mineral osseux en fonction de l'age. Nouv. Presse Med., 10: 3831 (1981).

11. Dickson, I. R., Miller, E. A., and Veis, A.: Evidence for abnormality of bonematrix proteins in osteogenesis imperfecta. Lancet, 2: 586 (1975).
12. Doty, S. B. and Mathews, R. S.: Electron microscopic and histochemical investigation of osteogenesis imperfecta tarda. Clin. Orthop. Rel. Res., 80: 191 (1971).

13. Eastoe, J. E., Martens, P. and Thomas, N. R.: The amino acid composition of human hard tissue collagens in osteogenesis imperfecta and dentinogenesis imperfecta. Calcif. Tiss. Res., 12: 91 (1973).

14. Falvo, K. A. and Bullough, P. G.: Osteogenesis imperfecta: A histometric analysis. J. Bone Joint Surg., 55A: 275 (1973).

15. Follis, R. H.: Osteogenesis imperfecta congenita: A connective tissue diathesis. J. Pediatr., 41: 713 (1952).

16. Frost, H. M.: Dynamics of bone remodeling. In : H.M. Frost, Ed.: Bone Biodynamics. pp. 328-354 (Little, Brown and Co., Boston, MA, 1964).

17. Frost, H. M.: Tetracycline based histological analysis of bone remodeling. Calcif. Tiss. Res., 3: 211 (1969).

18. Frost, H. M.: A method of analysis of trabecular bone dynamics. IN: P.J. Meunier, Ed.: Bone Histomorphometry. pp. 445-476 (Lab Armour Montagu, Paris, France, 1977).

19. Glorieux, F. H., Marie, P. J., Pettifor, J. M., and Delvin, E. E.: Bone response to phosphate salts, ergocalciferol and calcitriol in hypophosphatemic vitamin D resistant rickets. N. Engl. J. Med., 303: 1023 (1980).

20. Potts, J. $\Upsilon$. and Krutzik, S.: Parathyroid hormone. In: A.L. Nichols and J. Nelson, Eds., 4th Edition: Radioimmunoassay Manual. pp. 105-109 (Nichols Institute, Los Angeles, CA, 1977).

21. Kirivikko, K. I.: Urinary excretion of hydroxyproline in health and disease. Int. Rev. Conn. Tiss. Res., 5: 93 (1970).

22. Luciani, J. C., Ferran, J. L., Dumas, M. L., Meunier, P. J., and Dumas, R.: Osteodystrophic chez l'enfant hemodialyse. Nouv. Presse Med., 6: 3615 (1977).

23. Marie, P. J. and Glorieux, F. H.: Histomorphometric study of bone remodeling in vitamin D resistant rickets. Metab. Bone Dis. Rel. Res., 3: 31 (1981).

24. Marie, P. J. and Glorieux, F. H.: Personal communication.

25. Parekh, A. C. and Jung, D. H.: An improved method for the determination of hydroxyproline in urine. Biochem. Med., 4: 446 (1970).

26. Ramser, J. R., Villanueva, A. R., Pirok, D., and Frost, H. M.: Tetracycline based measurement of bone dynamics in three women with osteogenesis imperfecta. Clin. Orthop. Rel. Res., 49: 151 (1966).

27. Remigio, P. A. and Grynvalsky, H. T.: Osteogenesis imperfecta congenita: Association with conspicuous extraskeletal connective tissue dysplasia. Am. J. Dis. Child., 119: 524 (1970).

28. Riley, F. C., Jowsey, J., and Brown, D. M.: Osteogenesis imperfecta: Morphologic and biochemical studies of connective tissue. Pediatr. Res., 7: 757 (1973).

29. Robichon, J. and Germain, J. P.: Pathogenesis of osteogenesis imperfecta. Canad. Med. Assoc. J., 99: 975 (1968).

30. Schoenfeld, Y. A., Fried, A., and Ehrenfeld, N. E.: Osteogenesis imperfecta. Am. J. Dis. Child., 129: 679 (1975).

31. Sillence, D. O., Senn, A., and Danks, D. M.: Genetic heterogeneity in osteogenesis imperfecta. J. Med. Genet., 16: 101 (1979).

32. Shepard, R. M., Horst, R. L., Hamstra, A. J., and DeLuca, H. F.: Determination of vitamin $\mathrm{D}$ and its metabolites in plasma from normal and anephric man. Biochem. J., 182: 55 (1979).

33. Trelstad, R., Rubin, D., and Gross, J.: Osteogenesis imperfecta congenita: Evidence for a generalized molecular disorder of collagen. Lab. Invest., 36: 501 (1977).

34. Requests for reprints should be addressed to: Dr. Roland Baron, Department of Internal Medicine, Yale University School of Medicine, 333 Cedar Street, New Haven, CT 06510

35. This research was supported by Grant RR 00125 to the Yale Childrens Clinical Research Center. Doctor Gertner was supported by a Clinical Associate Physician Award from the Division of Research Resources.

36. The authors are very grateful to Doctors $\mathbf{F}$. Glorieux (Montreal) and $M$. Norman (Philadelphia) for providing biopsies from normal children and to Doctor $\mathbf{P}$. Marie (Montreal) for providing us with normal data for mineralization rate in children. They also want to thank Mrs. Lynn Neff for her technical help and Mrs. Marie Silberberg for typing the manuscript.

37. Received for publication March 4, 1982.

38. Accepted for publication June 18, 1982. 